Actes des congrès de la Société française

Shakespeare Shakespeare

17| 1999

Shakespeare et la voix

\title{
Rousseau et la parole politique
}

\section{Pierre Manent}

\section{(2) OpenEdition \\ Journals}

Édition électronique

URL : http://journals.openedition.org/shakespeare/422

DOI : 10.4000/shakespeare.422

ISSN : 2271-6424

Éditeur

Société Française Shakespeare

Édition imprimée

Date de publication : 1 novembre 1999

Pagination : 187-192

ISBN : 2-84269-331-0

Référence électronique

Pierre Manent, «Rousseau et la parole politique », Actes des congrès de la Société française Shakespeare [En ligne], 17 | 1999, mis en ligne le 01 novembre 2007, consulté le 01 mai 2019. URL : http:// journals.openedition.org/shakespeare/422 ; DOI : 10.4000/shakespeare.422 


\section{S H A K E S P E A R E \\ \& $\quad$ L $A \quad$ V O I X}

Société Française Shakespeare

Actes du Congrès de 1999

米米

Textes réunis et présentés par

Patricia DORVAL

publiés sous la direction de

Jean-Marie MAGUIN 


\section{ROUSSEAU ET LA PAROLE POLITIQUE}

Rousseau est peut-être le seul philosophe politique musicien. Il fut prodigieusement attentif à toutes les voix : voix du Peuple et voix de Dieu, surtout voix de la Nature. C'est la nature qui parle, mais aussi c'est la voix qui est naturante, qui fait advenir la nature de l'homme... Dans la cité bien ordonnée, l'homme peut parler, faire entendre sa voix, dire une parole.

Dans le Contrat Social, Rousseau écrit ceci : «Chez les Grecs tout ce que le peuple avait à faire il le faisait par lui-même ; il était sans cesse assemblé sur la place. Il habitait un climat doux, il n'était point avide, des esclaves faisaient ses travaux, sa grande affaire était sa liberté. N'ayant plus les mêmes avantages, comment conserver les mêmes droits? Vos climats plus durs vous donnent plus de besoins, six mois de l'année la place publique n'est pas tenable, vos langues sourdes ne peuvent se faire entendre en plein air, vous donnez plus à votre gain qu'à votre liberté, et vous craignez bien moins l'esclavage que la misère. [...] Pour vous, peuples modernes, vous n'avez point d'esclaves, mais vous l'êtes; vous payez leur liberté de la vôtre. Vous avez beau vanter cette préférence, j'y trouve plus de lâcheté que d'humanité» (III.15).

Dans cette vignette prodigieusement expressive, Rousseau décrit l'expérience grecque de la parole publique. Il la dispose comme le rideau de la scène historique sur lequel nous pouvons lire la dégradation et la corruption modernes. Celles-ci appellent deux chefs d'accusation : 
- notre «liberté» si vantée nous assujettit à l'économie ; dans une société fondée sur l'intérêt, nous sommes esclaves du système des besoins : Rousseau ici anticipe Marx.

- notre «liberté» si vantée est une illusion complaisante; nous n'avons plus le courage d'avoir des esclaves pour être vraiment libres : Rousseau ici anticipe Nietzsche.

Ces implications critiques sont moins intéressantes pour nous que la proposition positive, que le modèle grec. En quoi consiste-til ? Qu'est-ce qui en fait précisément un modèle ? On répond : la cité grecque, c'est la démocratie directe. Cela est vrai, mais reste un peu abstrait. Rousseau dit plus simplement : dans la cité grecque, on peut parler et s'entendre. Ce qui définit la cité désirable, ce sont les conditions de la parole audible. On est frappé par le contraste entre la simplicité apparente de son propos et l'extrême complexité des analyses et propositions des philosophes contemporains qui se donnent apparemment la même tâche, voulant élaborer les conditions de la communication démocratique. Rousseau est indifférent à toutes ces conditions transcendantales. Il pose seulement une condition réelle, ou deux conditions réelles qui n'en font qu'une : que les citoyens parlent en personne et qu'ils soient audibles.

En un sens, Rousseau ne fait que reprendre la thèse des philosophes grecs eux-mêmes, celle d'Aristote en particulier qui insiste sur la nécessité pour la cité d'être de dimensions modestes afin que les citoyens puissent se voir et s'entendre: que tous les citoyens soient visibles, que toutes leurs paroles soient audibles! D'ailleurs Aristote définit équivalemment l'homme comme l'animal politique et comme l'animal ayant le logos, soit la parole.

Mais le propos de Rousseau n'est pas la simple répétition du propos d'Aristote. Rousseau est le témoin d'une aventure de la langue. Quelque chose est arrivé aux langues modernes, qui change les conditions de l'expérience politique et humaine. Le problème des peuples modernes, c'est qu'ils parlent des langues que l'on n'entend pas. Et à la transformation de la langue correspond une transformation politique et anthropologique.

A l'origine de la parole humaine, il y a l'amour, explique Rousseau dans l'Essai sur l'origine des langues. Les jeunes filles et les jeunes hommes se rencontrent autour des points d'eau: «en s'efforçant de se faire entendre on apprit à s'expliquer. [...] Là fut enfin le vrai berceau des peuples, et du pur cristal des fontaines sortirent les premiers feux de l'amour» ${ }^{1}$. Cette parole amoureuse, 
cette première parole expressive fit sortir l'humanité de l'inceste primitif : «Quoi donc ! avant ce temps les hommes naissaient-ils de la terre? Les générations se succédaient-elles sans que les deux sexes fussent unis et sans que personne s'entendît? Non, il y avait des familles, mais il n'y avait point de nations; il y avait des langues domestiques, mais il n'y avait point de langues populaires ; il y avait des mariages, mais il n'y avait point d'amour. Chaque famille se suffisait à elle-même et se perpétuait par son seul sang. Les enfants nés des mêmes parents croissaient ensemble et trouvaient peu à peu des manières de s'expliquer entre eux; les sexes se distinguaient avec l'âge, le penchant naturel suffisait pour les unir, l'instinct tenait lieu de passion, l'habitude tenait lieu de préférence, on devenait maris et femmes sans avoir cessé d'être frère et soeur. Il n'y avait là rien d'assez animé pour dénouer la langue.... ${ }^{2}$. En faisant sortir hommes et femmes de la caverne des Cyclopes, la parole amoureuse - la voix qui chante et persuade fait advenir l'humanité proprement dite.

Rousseau pose une équivalence, et même une identité, entre la parole amoureuse et la parole humaine originelle, parce que la parole amoureuse est essentiellement expressive, idéalisante, enthousiaste : elle traduit un transport et elle transporte. Mais la parole expressive, idéalisante, peut traduire et susciter d'autres transports, d'autres enthousiasmes, en particulier politiques ou religieux. Ainsi, peu après avoir évoqué l'idylle autour des puits, Rousseau décrit en ces termes la parole politique et religieuse de Mahomet : «Tel pour savoir lire un peu d'arabe sourit en feuilletant l'Alcoran, qui, s'il eût entendu Mahomet l'annoncer en personne dans cette langue éloquente et cadencée, avec cette voix sonore et persuasive qui séduisait l'oreille avant le cœur, et sans cesse animant ses sentences de l'accent de l'enthousiasme, se fût prosterné contre terre en criant, grand Prophète Envoyé de Dieu, Menez-nous à la gloire, au martyre; nous voulons vaincre ou mourir pour vous ${ }^{3}$. En tout cas, amoureuse, politique ou religieuse, la parole authentique met celui qui l'entend hors de lui.

L'ordre humain originel, c'est cet ordre expressif, c'est l'ordre traduit et gouverné par la voix humaine que l'on entend et qui emporte. Mais aujourd'hui..., aujourd'hui la langue mélodieuse des Grecs a été perdue ${ }^{4}$. Nos langues sont les langues sourdes du nord, non plus langues de l'amour et de la passion, mais langues de l'intérêt et de la raison : «Le français, l'anglais, l'allemand sont le langage privé des hommes qui s'entr'aident, qui raisonnent entre eux de sang-froid $[\ldots] \gg^{5}$. 
La langue que nous parlons, langue de «peuples commerçants» ${ }^{6}$, qui concerne principalement les besoins tels que l'intérêt les éprouve et que la raison les constate, est une langue qui pourrait se contenter de gestes et de signes : «si nous n'avions jamais eu que des besoins physiques, nous aurions fort bien pu ne parler jamais et nous entendre parfaitement par la seule langue du geste. Nous aurions pu établir des sociétés peu différentes de ce qu'elles sont aujourd'hui, ou qui même auraient marché mieux à leur but $[\ldots] \gg 7$. Ainsi les peuples modernes se servent d'une langue qui, par ses restes d'expressivité, leur est au fond inutile. Rousseau aurait certainement trouvé la confirmation de son diagnostic dans les efforts que nous avons déployés en ce siècle pour parvenir à élaborer des langages purement rationnels, c'est-à-dire dépourvus de toute expressivité, et pour autant exactement adéquats à nos besoins sociaux! Il suffit de mentionner le calcul économique, la philosophie analytique, la communication électronique. Quant à la «langue blanche», comme on dit la «voix blanche», d'une certaine littérature contemporaine, qu'est-elle d'autre que l'effort pour tirer des effets expressifs d'une langue que l'on veut rigoureusement sans expression?

Pourquoi un changement si profond des conditions de la parole humaine? Il provient selon Rousseau d'un double développement, ou du développement simultané et corrélatif de la force publique et de l'intérêt privé - de l'État et de la société. La société - le système des besoins - se déploie, s'étend, se complexifie en même temps que l'État - «absolu» puis «représentatif» - s'élève audessus de la société. A la cité gouvernée par une parole expressive, une voix que l'on entend, succède un ordre politique divisé et articulé entre la société et l'État qui la représente. Mais qui parle dans cet ordre nouveau, qui parle vraiment ? Plus personne ne parle, semble-t-il, plus personne ne parle vraiment! La société ne parle pas puisqu'elle a confié sa parole la plus ample à l'État qui la représente : elle a autorisé l'État à parler pour elle. Mais ce n'est pas non plus l'État qui parle vraiment, puisqu'il n'a pas de parole qui lui soit propre: il n'est que le porte-parole de la société. L'inexpressivité du système représentatif oblige à inventer un mécanisme compliqué pour compter les voix que l'on n'entend plus.

Ces remarques suggèrent ce que fut, dans ses grandes lignes, l'histoire de la parole politique en Occident. Les républiques anciennes étaient parlantes et audibles. Les monarchies anciennes étaient parlantes et audibles. A partir de Louis XIV, la voix du Roi 
ne fut plus audible. On lui construisit un théâtre où il ne parle plus que pour quelques auditeurs et spectateurs: Versailles. La monarchie devient un théâtre où le peuple n'a pas accès, et, symétriquement, c'est dans le théâtre que se réfugie la parole vive qui intéresse le peuple : «Depuis longtemps on ne parle plus au public que par les livres, et si on lui dit encore de vive voix quelque chose qui l'intéresse, c'est au théâtre» ${ }^{8}$. D'où l'ambiguiité du théâtre, à la fois lieu corrupteur puisqu'il rend l'homme spectateur passif de son humanité, ainsi que la Lettre à d'Alembert l'explique de manière si impressionnante, mais peut-être aussi lieu salvateur ou du moins correcteur, puisqu'il est le refuge d'une parole expressive dans une société qui l'est de moins en moins. Mais comment le théâtre pourrait-il rester expressif dans une société qui perd de plus en plus le sens de la parole vive? Rousseau nous suggère que le théâtre est politiquement de plus en plus nécessaire à mesure qu'il est expressivement de moins en moins possible.

Pierre MANENT

Centre de Recherches Politiques Raymond Aron École des Hautes Études en Sciences Sociales

\section{NOT E S}

${ }^{1}$ Ducros, éd., 1970, p. 123.

${ }^{2}$ Souligné par moi, lbid., p. 125.

${ }^{3}$ Ibid., p. 137.

${ }^{4}$ Ibid., p. $141 \& 143$.

${ }^{5}$ Ibid., p. 135

${ }^{6}$ Ibid., p. 57.

${ }^{7}$ Ibid., p. 35 \& 37.

${ }^{8}$ Ibid., p. 218. 